\title{
Gestational gigantomastia complicated with mastitis
}

\author{
Calina Maria Loures de Oliveira Teixeira' (D), Carolina Teixeira de Assis Lopes² (1),
}

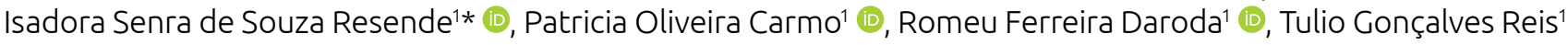

\section{ABSTRACT}

Gestational gigantomastia (GG) is a disease defined by rapid and disproportionate breastenlargement in pregnancy. Its complications may lead to emergency mastectomy or induced miscarriage. We present a case report in which pregnancy had a favorable evolution after the surgical intervention of the breasts.

KEYWORDS: Breast; Pregnancy; Hypertrophy; Mastitis; Mastectomy.

\section{INTRODUCTION}

Gestational gigantomastia (GG) is a disease defined by rapid and disproportionate breast enlargement during pregnancy. Its complications may lead to emergency mastectomy or induced miscarriage $e^{1-4}$.

With few reports in the literature, its incidence varies from one in 28 thousand to one in 100 thousand pregnancies worldwide, so it is very rare and has unpredictable progression. The etiology and pathogenesis remain uncertain, but the most accepted theories are hormonal and autoimmune $\mathrm{e}^{1-3}$.

The previous history is the strongest risk factor for its recurrence, and, despite the benignity, the clinical presentation can simulate malignancy and should be excluded initially. As a treatment, conservative modalities are ineffective, justifying surgical interventions, especially total mastectomy ${ }^{1,5}$.

\section{CASE REPORT}

Patient R.S.C.A., 34 years old, secundigravida, 9-10 weeks of gestational age, chronic hypertension, sought medical attention at the emergency department complaining of mastalgia and increased breast volume. On physical examination, she presented extremely swollen and hyperemic breasts, palpating poorly defined tumors, the largest of which was $5 \mathrm{~cm}$ in the upper quadrant of the left breast (Figure 1).

She was admitted to the obstetrics service of the institution with a diagnosis of mastitis, and treated with intravenous antibiotic therapy with cephalothin and metronidazole, oral analgesia, and the doctors provided direction on general breast care were started. The presence of thickening of the subcutaneous tissue was confirmed, with blurring of the adjacent fat, inferring an inflammatory and/or infectious process without an organized collection, associated with the presence of bulky solid hypoechoic nodules bilaterally, suggestive of fibroadenomas - Breast Imaging Reporting and Data System (BI-RADS) category 3 ultrasound.

A core biopsy guided by ultrasound was performed in the following month, whose anatomopathology revealed benign glandular breast tissue associated with a non-specific mild chronic inflammatory process. The immunohistochemical report showed negativity for neoplasm, which was observed in a negative expression of the c-erbB-2 oncoprotein correlated with a positive expression of the other antibodies. Complementary magnetic resonance imaging was not possible due to the technical difficulty caused by breast volume.

R.S.C.A. was readmitted after 40 days of core biopsy, showing an increase in breast volume with the presence of phlogistic signs (hyperemia, hyperthermia) and extensive ulcerated lesion in lower quadrants of the right breast (puncture site), without a foul odor (Figure 2). Armpits and supraclavicular fossae were free. A biopsy of the ulcerated area was performed, showing only ulceration, chronic inflammatory infiltrate, and granulation tissue and material were also sent for polymicrobian culture, which was positive for infection by multisensitive Acinetobacter baumannii. Vancomycin and meropenem were then started, maintained for seven days. Organic lesions were absent, but anemia was maintained, and inflammatory tests were touched in the laboratory control.

'Santa Casa de Misericórdia de Juiz de Fora - Juiz de Fora (MG), Brazil.

IInstituto Oncológico de Juiz de Fora - Juiz de Fora (MG), Brazil.

*Corresponding author: isadorassresende@gmail.com

Conflict of interests: nothing to declare.

Received on: 01/10/2020. Accepted on: 08/06/2020 
This outcome proved to be decisive in the team's decision, which was demonstrated by the failure in the usual clinical treatment of recurrent mastitis, in addition to the possible risks associated with maternal and fetal life. Due to the rarity of the pathology and the unfavorable progression of the clinical condition, despite the probable benignity until that moment, and prioritizing maternal-fetal well-being, after a broad multidisciplinary discussion, the surgical resolution of the condition was chosen, with an informed consent form signed by the patient and team. The patient underwent a simple bilateral mastectomy with removal of both breasts. The right weighed 7,660 grams and the left 4,960 grams, with drainage through a bilateral suction drain. She received red blood cells transfusions and presented a positive fetal heart rate (FHR) of $156 \mathrm{bpm}$, regular at the end of the

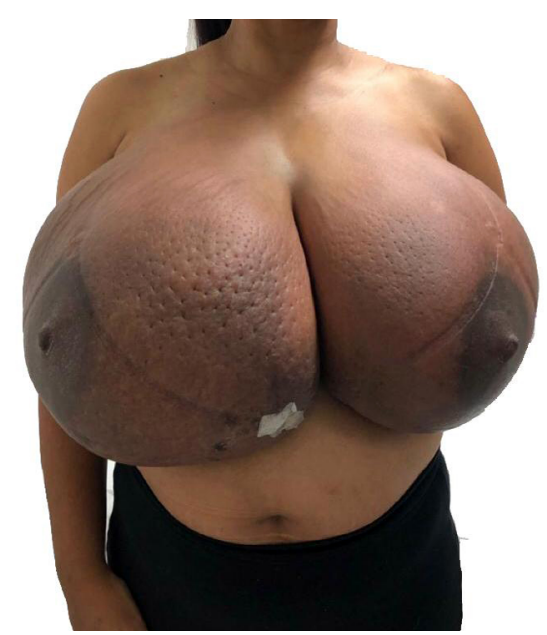

Figure 1. 34-year-old patient, presenting bilateral breast volume enlargement, extensive edema, and hyperemia, palpating poorly defined tumors.

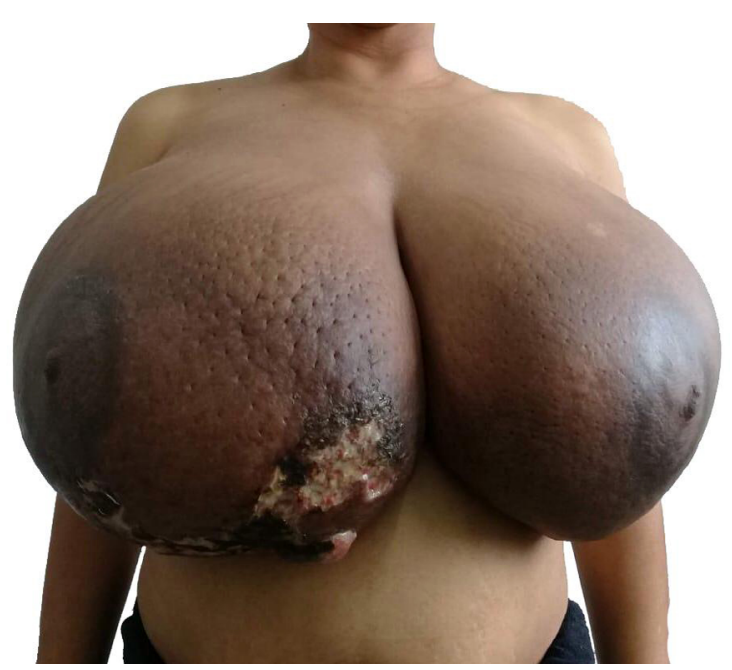

Figure 2. Increased breast volume, with the presence of phlogistic signs (hyperemia, hyperthermia) and extensive ulcerated lesion in the lower quadrants of the right breast. procedure, and absence of vaginal bleeding. The anatomopathological examination resulting from the procedure corroborated the primary findings of benignity, showing extensive lobular hyperplasia and ulcerated lesion associated with an inflammatory process. No other particularities on skin or nipple were shown.

At 38 weeks of gestational age, the patient was referred from prenatal care for pregnancy resolution due to a hypertensive peak $(150 \times 100 \mathrm{mmHg})$. After performing the pre-eclampsia routine laboratory evaluation and obstetric Doppler ultrasound - all exams without changes -, the labor induction was started with misoprostol administered vaginally. The patient progressed to vaginal delivery, on January 12, 2019, a female newborn, remaining in joint accommodation in the puerperium and being discharged in excellent clinical conditions, accompanied by her daughter. Future mammoplasty with bilateral breast prosthesis placement is scheduled.

\section{DISCUSSION}

GG or gravida macromastia is defined as a disorder characterized by diffuse, extreme, and disabling enlargement of one or both breasts during pregnancy, which was first described in 1648 by Palmuth $^{1,2,6}$. Its etiology is still unknown ${ }^{1,7,8}$.

The main associated physical symptoms are: breast pain, infection, ulceration, postural problems, back pain, and even postural instability. As a result of an infection not treated properly, it can progress to severe sepsis, kidney dysfunction, multiple organ dysfunction syndrome, and even death. Besides the physical problems, it can cause social, emotional, and psychological problems for women affected ${ }^{1,5,6}$.

The condition is mostly found in caucasians, multiparous, with autoimmune diseases, with no association with age or fetal gender. It can occur in any pregnancy; however, the previous history is the strongest risk factor for its recurrence, as well as the patient being submitted to mammoplasty instead of total mastectomy ${ }^{1,5,7}$. Most cases of GG are bilateral and begin in the first trimester or at the beginning of the second, coinciding with the peak period of gonadotropin production during pregnancy, which further strengthens the hypothesis of hormonal association ${ }^{1-3,6}$.

The differential diagnosis includes phyllodes tumor, fibroadenoma, and lymphomas, which must be excluded by biopsy and immunohistochemistry. Histologically, the breast tissue of patients with GG presents significant lobular hypertrophy, ductal proliferation, and periductal fibrosis ${ }^{1-3,6}$. Histological and laboratory markers of autoimmunity can also be tested, although they have not been performed in the case described ${ }^{5-7}$. Despite the benignity of GG, the clinical presentation - rapid breast enlargement, edema of the underlying tissue, the appearance of the tissue, bilateral axillary edema - can simulate malignancy and, therefore, this should be excluded initially,6. 
Treatment for GG is multiple: conservative, pharmacologi$\mathrm{cal}$, and surgical ${ }^{5}$. Several authors propose to use bromocriptine, despite the variable and generally temporary effects, with surgery being the basis of treatment ${ }^{4}$. Such medication is safe during pregnancy, although reports of isolated cases suggest delayed intrauterine growth as an isolated side effect. Therefore, it is recommended that serial fetal growth monitoring be performed in patients on bromocriptine for gigantomastia ${ }^{2}$. Although an attempt at drug treatment should be made in all patients, surgery is the treatment basis. Given the risk that surgeries will induce premature births, this should be done to postpone the surgery to a stage in which there is a viable fetus. A good indication of anticipating the surgical intervention period, in this context, would be the mastitis complication, for which the failure in immediate antibiotic therapy directed by culture and sensitivity implies obstetric risks ${ }^{8-10}$. Two main surgical modalities that have been widely used in the treatment are mammoplasty and total mastectomy $y^{9}$. As there is a possibility of recurrence with mammoplasty, bilateral mastectomy with reconstruction is the treatment of choice in women who want future pregnancies ${ }^{1-3,6}$.

In the past, it was customary to recommend elective pregnancy termination in patients with GG. This is definitely not relevant in today's world, due to the evolution of Medicine, anesthesia, and surgery. The care that was mostly conservative is being discouraged, and surgical treatment of this condition is chosen even during pregnancy ${ }^{1,5}$.

\section{CONCLUSION}

GG is a benign condition that can simulate carcinomatosis, with unpredictable, markedly rapid, and progressive evolution. Its association with mastitis is a rare presentation, for which multidisciplinary efforts must be considered in breast and fetal preservation. In the presence of an unfavorable evolution for mastitis, mastectomy should be considered, aiming at maternal preservation and fetal health.

\section{AUTHORS' CONTRIBUTIONS}

C.M.L.O.T.: conceived the present idea, conceptualization, data curation, formal analysis, investigation, visualization, writing - original draft, writing - review \& editing.

C.T.A.L.: conceived the present idea, conceptualization, data curation, formal analysis, visualization, writing — original draft, writing - review \& editing.

I.S.S.R.: conceived the present idea, conceptualization, data curation, formal analysis, visualization, writing - original draft, writing - review \& editing.

P.O.C.: conceptualization, data curation, formal analysis, investigation, visualization, writing - original draft.

R.F.D.: conceptualization, data curation, visualization, writing - original draft.

T.G.R.: conceived the present idea, conceptualization, data curation, formal analysis, visualization, writing — original draft.

\section{REFERENCES}

1. Mangla M, Singla D. Gestational Gigantomastia: A Systematic Review of Case Reports. J Mid-life Health. 2017;8(1):40-4. https://dx.doi.org/10.4103\%2Fjmh.JMH_92_16

2. Bukhari SS, Manan H, Khan MM, Raza SS. Resolution Of Gestational Gigantomastia With Termination Of Pregnancy. J Ayub Med Coll Abbottabad. 2018;30(2):298-300.

3. Kaluarachchi A, Rishard MRM, Ranaweera AKP, Beneragama T, Silva MVC. Gestational gigantomastia complicated with deep vein thrombosis. Ceylon Med J. 2018;63(2):84-5.

4. Maluf Junior I, Freitas RS, Budel VM, Amaro LC, Scomação I, Roca RB, et al. Gigantomastia gestacional: como abordar essa situação clínica. Rev Bras Cir Plást. 2015;30(1):134-7. http:// www.dx.doi.org/10.5935/2177-1235.2015RBCP0129

5. Rezai S, Nakagawa JT, Tedesco J, Chadee A, Gottimukkala S, Mercado R, et al. Gestational Gigantomastia Complicating Pregnancy: A Case Report and Review of the Literature. Case Rep Obstet Gynecol. 2015;2015:892369. https://doi. org/10.1155/2015/892369
6. Türkan H, Gökgöz MS, Ta冈delen I, Dündar HZ. Gestational Gigantomastia. J Breast Health. 2016;12(2):86-7. https://dx.doi. org/10.5152\%2Ftjbh.2016.2852

7. Touraine P, Youssef N, Alyanakian MA, Lechat X, Balleyguier C, Duflos C, et al. Breast Inflammatory Gigantomastia and Autoimmunity. J Clin Endocrinol Metabol. 2005;90(9):5287-94. https://doi.org/10.1210/jc.2005-0642

8. Zhou M, Jin M, Wang L, Pan LJ. Pregnancy-associated gigantomastia recurrence and ectopic breast after reduction mammaplasty: A case report. Cancer Biomarkers. 2017;20(2):225-9. https://doi.org/10.3233/cbm-160450

9. Antevski BM, Smilevski DA, Stojovski MZ, Filipovski VA, Banev SG. Extreme gigantomastia in pregnancy: case report and review of literature. Arch Gynecol Obstet. 2007;275(2):14953. https://doi.org/10.1007/s00404-006-0190-7

10. CanterJW,Oliver GC,ZaloudekCJ.SurgicalDiseases oftheBreast During Pregnancy. Clin Obstet Gynecol. 1983;26(4):853-64. https://doi.org/10.1097/00003081-198312000-00010 